\title{
The effectiveness and safety of paediatric prehospital pain management: a systematic review
}

Yonas Abebe ${ }^{1,2^{*}} \mathbb{0}$, Fredrik Hetmann², Kacper Sumera ${ }^{3}$, Matt Holland ${ }^{4}$ and Trine Staff ${ }^{2}$

\begin{abstract}
Background: Clinically meaningful pain reduction with respect to severity and the adverse events of drugs used in prehospital pain management for children are areas that have not received sufficient attention. The present systematic review therefore aims to perform a comprehensive search of databases to examine the preferable drugs for prehospital pain relief in paediatric patients with acute pain, irrespective of aetiology.
\end{abstract}

Methods: The systematic review includes studies from 2000 and up to 2020 that focus on children's prehospital pain management. The study protocol is registered in PROSPERO with registration no. CRD42019126699. Pharmacological pain management using any type of analgesic drug and in all routes of administration was included. The main outcomes were (1) measurable pain reduction (effectiveness) and (2) no occurrence of any serious adverse events. Searches were conducted in PubMed, Medline, Embase, CINAHL, Epistemonikos and Cochrane library. Finally, the risk of bias was assessed using the Joanna Briggs Institute (JBI) checklist and a textual narrative analysis was performed due to the heterogeneity of the results.

Results: The present systematic review on the effectiveness and safety of analgesic drugs in prehospital pain relief in children identified a total of eight articles. Most of the articles reviewed identified analgesic drugs such as fentanyl (intranasal/IV), morphine (IV), methoxyflurane (inhalational) and ketamine (IV/IM). The effects of fentanyl, morphine and methoxyflurane were examined and all of the included analgesic drugs were evaluated as effective. Adverse events of fentanyl, methoxyflurane and ketamine were also reported, although none of these were considered serious.

Conclusion: The systematic review revealed that fentanyl, morphine, methoxyflurane and combination drugs are effective analgesic drugs for children in prehospital settings. No serious adverse events were reported following the administration of fentanyl, methoxyflurane and ketamine. Intranasal fentanyl and inhalational methoxyflurane seem to be the preferred drugs for children in pre-hospital settings due to their ease of administration, similar effect and safety profile when compared to other analgesic drugs. However, the level of evidence (LOE) in the included studies was only three or four, and further studies are therefore necessary.

Keywords: Children, Paediatrics, Prehospital, Ambulance, Analgesia, Pain management, Fentanyl, Morphine, Methoxyflurane, Ketamine

*Correspondence: yonbbb@gmail.com; yonasabe@oslomet.no 1 Department of Emergency and Critical Care Nursing, St. Paul's Hospital Millennium Medical College, Addis Ababa, Ethiopia

Full list of author information is available at the end of the article

\section{Background}

Prehospital care providers have traditionally focused on time-sensitive acute illness and major traumas, which represent only a small number of patients. However, in original author(s) and the source, provide a link to the Creative Commons licence, and indicate if changes were made. The images or other third party material in this article are included in the article's Creative Commons licence, unless indicated otherwise in a credit line to the material. If material is not included in the article's Creative Commons licence and your intended use is not permitted by statutory regulation or exceeds the permitted use, you will need to obtain permission directly from the copyright holder. To view a copy of this licence, visit http://creativecommons.org/licenses/by/4.0/. The Creative Commons Public Domain Dedication waiver (http://creativeco mmons.org/publicdomain/zero/1.0/) applies to the data made available in this article, unless otherwise stated in a credit line to the data. 
the prehospital setting, large groups of patients experience a variety of illnesses and injuries with frequent symptoms and signs, including pain [1]. It is thought that effective analgesia is one of the top outcome measures of prehospital care [2]. It is also considered among the top factors in the satisfaction of the patients' family [3]. The prevalence of acute pain in prehospital settings ranges from 42 to $53 \%$ [4-6].

Previous studies of children in prehospital care show that although the pain was documented in the ambulance records, the level of pain was not assessed in $66-96 \%$ of cases $[5,7]$. Previous findings also show that $52-88 \%$ of children in prehospital setups did not receive pain medication despite having moderate to severe pain $[5,8]$. The most common reasons for not providing adequate pain medication to children in prehospital settings include fearing side effects, difficulties with intravenous (IV) line access, being under five years of age, the lack of a pain assessment and the assumption that children need less analgesia than adults [9-12].

The evidence, however, indicates that adequate prehospital pain management in children relieves suffering, contributes to timely emergency department (ED) analgesia, prevents chronic pain and improves recovery [13, 14]. Conversely, inadequate pain management harms children's development and increases morbidity and mortality $[15,16]$. The negative effect of inadequate pain management could also extend to fear of medical care or medical/medication over-use in adulthood [17].

In prehospital pain medication for children, clinically meaningful reductions of pain severity and evaluations of adverse events of drugs for different age groups are areas that lack sufficient investigation. A few small-scale and systematic reviews of prehospital pain management have been conducted, but these either concerned the adult age group, trauma aetiologies or were specific to one analgesic agent only [18-20]. Because of the lack of high-level evidence, there are no clear guidelines regarding the choice of drug, recommended dose or which route of administration is preferable for prehospital pain management of children, irrespective of aetiology [12, 21]. The current original systematic review aims to examine the effectiveness and safety of analgesic drugs for prehospital pain relief in paediatric patients with acute pain of any cause.

\section{Methods}

This systematic review was conducted in accordance with the Preferred Reporting Items for Systematic Reviews and Meta-analyses (PRISMA) guidelines [22]. The review question, outcomes, inclusion criteria and methods of analysis were predefined, and the protocol was registered in the International Prospective Register of
Systematic Reviews (PROSPERO), with registration no. CRD42019126699 [23].

\section{Review question and types of participants}

The main research question for this systematic review was, 'What are the preferred drugs for prehospital pain relief in paediatric patients with acute pain?'. The study participants were children under the age of eighteen with acute pain (i.e. a sudden pain lasting less than 3 months) in the prehospital setting. A prehospital setting is defined here as a place where any acute medical care is provided by ambulance care providers before the patient arrives at the hospital. In this review, preferable analgesic drugs were determined by the effectiveness and safety of the drugs. The primary outcome of this review concerned the effectiveness of the analgesic drugs employed. The drugs' effectiveness was defined as a clinically meaningful pain reduction as measured by a reduction of two or more points from the initial pain severity score after the administration of analgesic drugs based on standardised clinical pain assessment tools [24]. The secondary outcome concerned safety as defined by no occurrence of any serious adverse events in the prehospital setting after analgesic administration. The U.S. Food and Drug Administration (FDA) defines a serious adverse event as any serious undesirable experiences such as death, substantial risk of dying (life-threatening), hospitalisation, disability or permanent damage, congenital anomaly, required intervention to prevent permanent impairment and other serious medical events associated with the use of a medical product in a patient [25].

\section{Eligibility criteria}

The current review included all studies that focus on children's prehospital pain management in low-, middle- and high-income countries. We included any mixed age group studies if there was a separate analysis for children under eighteen years of age. Since knowing the standalone effect of other non-pharmacological pain managements is difficult, only studies that addressed pharmacological pain management with any type of analgesic drugs in all routes of administration were included. Pain evaluation papers were included if pharmacological pain interventions were integrated. To examine all types of analgesic drugs that are used, we included all qualifications of prehospital care providers despite variation in their training, scope and expertise in the different prehospital setups. The publications included in this review comprised randomised control trials, non-randomised control studies, a cohort with control groups, interrupted time series, cross-sectional and case series studies.

Studies that examined chronic pain were excluded because the patient's response to chronic pain 
management is different when compared to acute pain management [26]. As with the medical setup, the patients' characteristics and the level of the responders' training varied, studies that report on any sort of out-ofhospital pain treatments given by non-ambulance service providers, ambulance care while in medical transfers and in-hospital transfers were excluded. Pain management given in fixed healthcare facilities and/or by any other non-healthcare professionals was also excluded. As the primary and secondary outcomes of this review concerned the effectiveness and safety of analgesic drugs, studies that did not produce findings on either of these outcomes were excluded. In addition, qualitative studies, case reports, guidelines, continuing professional development (CPDs), letters to editors, service evaluation, conference abstracts and abstracts that did not have full text were also excluded.

\section{Search strategy}

The search strategy was developed by the four authors (YA, TS, FH and KS) who are the subject specialists and was peer-reviewed by another author $(\mathrm{MH})$ who is a research librarian. Studies were identified through electronic database searches including PubMed, Ovid Medline (1946 to 15 December 2020), Ovid Embase (1974 to 15 December 2000), CINAHL (Ebsco), Epistemonikos and Cochrane library. The relevant medical subject headings (MeSH terms) and keywords used for the systematic search strategy are presented in Table 1. The searches were limited as regards publication year (the past 20 years from 2000 to 2020) and language (English, Danish, Norwegian or Swedish). The search strategy for each database is presented in Tables 2, 3, 4, 5, 6, 7. All independent reviewers performed the literature searches from all included databases and imported these to the Covidence software. The last searches were rerun by the research librarian $(\mathrm{MH})$ on 16 December 2020. In addition, a hand search was conducted of the reference lists of the studies included in the current systematic review and systematic review reports concerning a similar topic, which resulted in the identification of one additional article.

\section{Study selection}

The results of the conducted search were combined, and duplicate studies were eliminated using the Covidence software. Titles and/or abstracts of studies were screened and carefully read by the four review authors (YA, TS, FH and KS) to identify potentially eligible studies. The full text of these articles was then retrieved and independently reviewed by the same four authors. The articles were included if the two authors agreed on that specific article. Discrepancies were resolved through discussion with a third reviewer (one of the four authors who had not reviewed that specific article).

\section{Data extraction}

We modified a data extraction template from Covidence version 2.0 [27]. One randomly selected article among the included studies was used as a pilot test. Two review authors performed the extraction (YA and TS) and resolved any disagreements through discussion. The general characteristics of the included studies, the name of the administered analgesic drug, the route of administration and the dose were extracted. In addition, the two outcome variables of the review and any age-specific results for small children ( $<5$ years old) were extracted. An email was forwarded to the respective corresponding authors of the included studies to obtain any additional information and confirm the correctness of the extracted data.

\section{Assessment of the risk of bias}

The Joanna Briggs Institute (JBI) checklist was used to assess the risk of bias for each of the study designs employed in the included articles [28]. The JBI checklists for analytical cross-sectional, prevalence, case series and cohort study designs were used. The level of evidence (LOE) was also classified according to the evidence evaluation worksheet by the International Liaison Committee on Resuscitation for therapeutic interventions (Table 8) [29]. Two authors (YA and KS) independently conducted a risk of bias assessment and a third author $(\mathrm{FH})$ resolved any disagreements. No article was excluded on the basis of these assessments.

\section{Data synthesis}

Due to the heterogeneity of outcome variables, we performed a textual narrative analysis of the findings from each of the included studies. We structured our synthesis based on the characteristics of the studies and the types of drugs they included.

\section{Results}

We imported a total of 10,844 studies from six electronic databases. After removing duplicates $(n=3696), 7,148$ titles/abstracts were screened. Of these, we reviewed 311 full-text studies. We also reviewed an additional 17 full-text studies identified from hand-searched reference lists. In all, 328 full-text articles comprising 320 studies were excluded, and eight studies [30-37] that met the eligibility criteria were included in the review. The selection process and grounds for exclusion are presented in a PRISMA flowchart below (Fig. 1). 


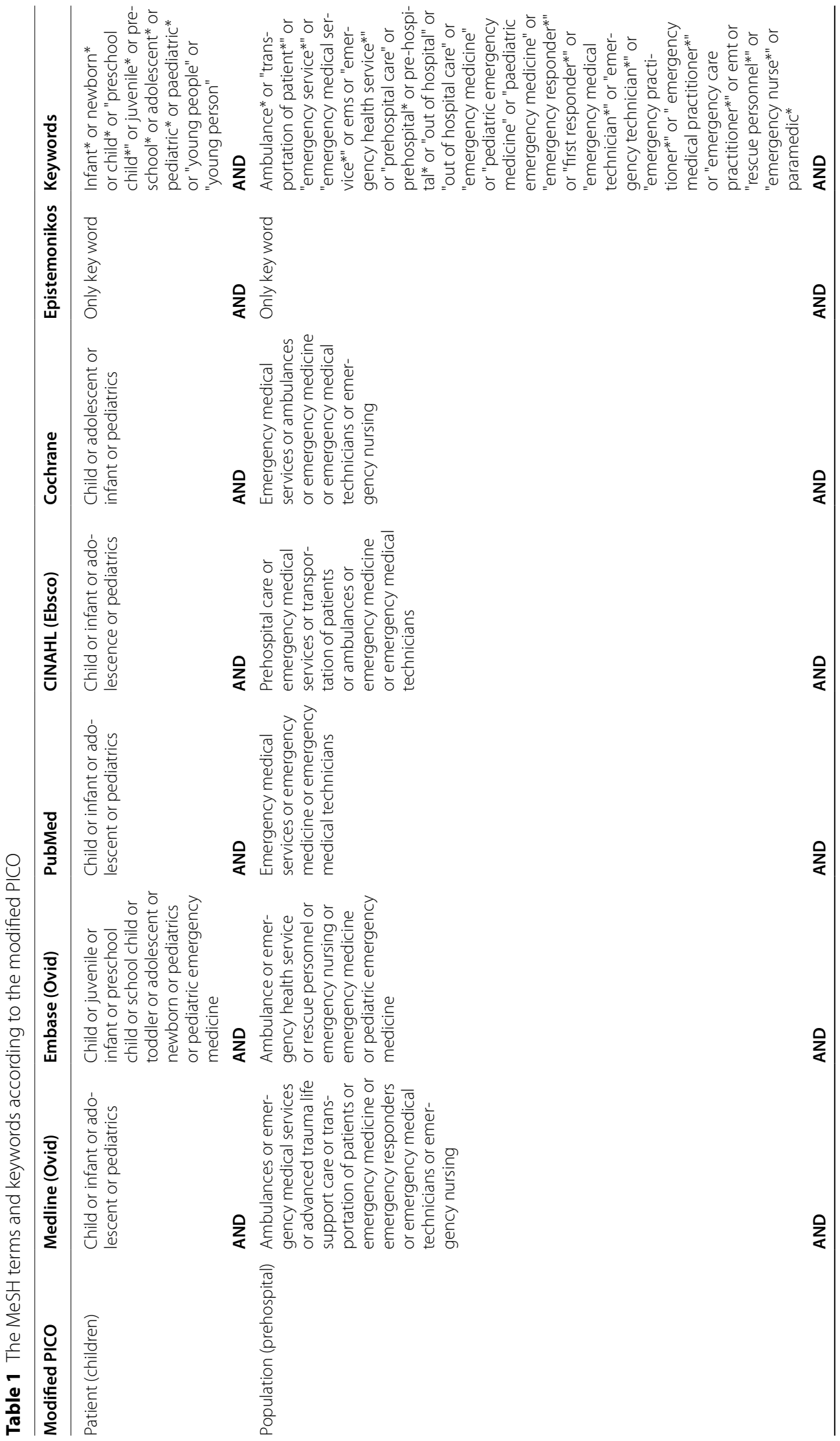




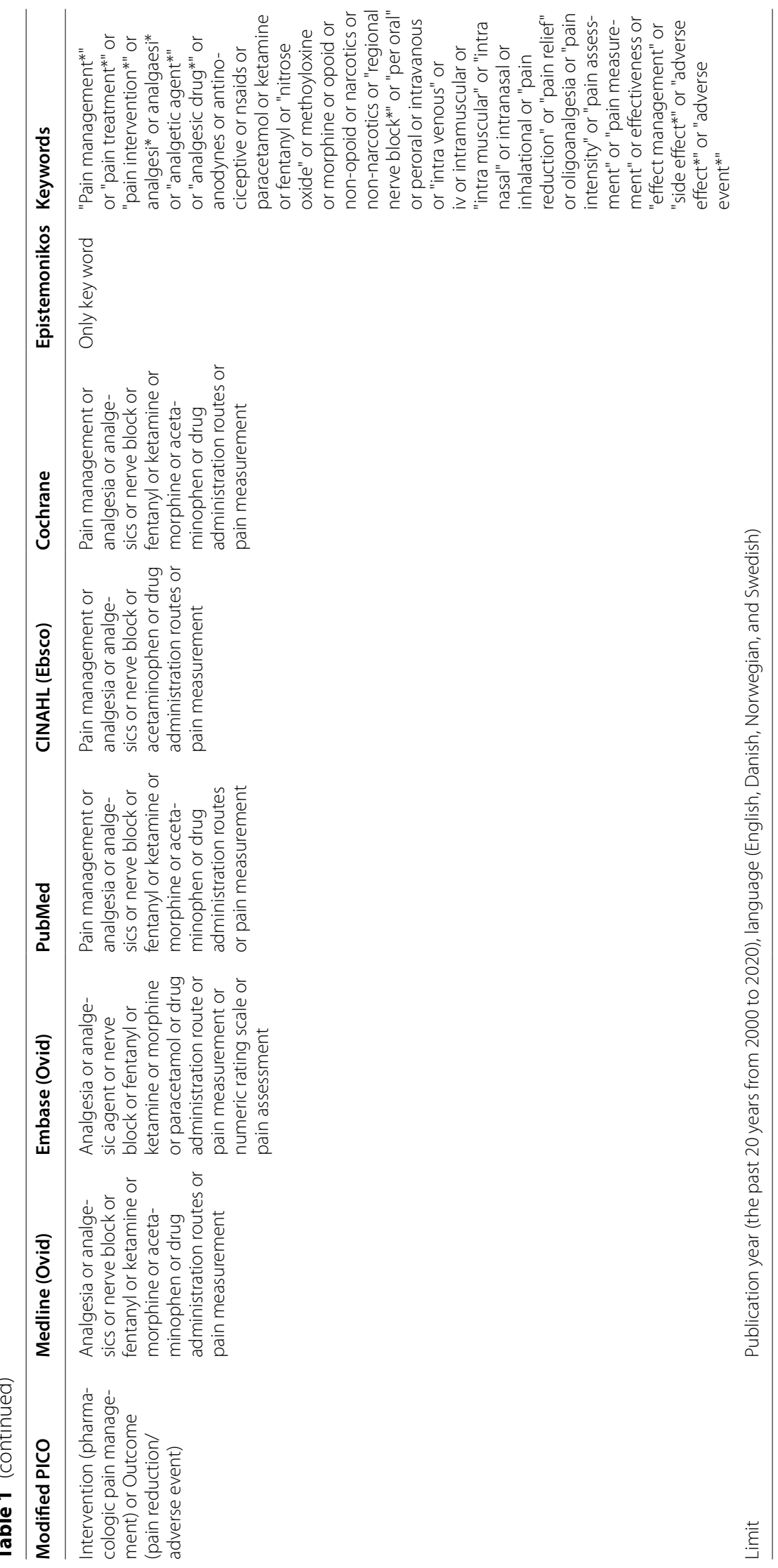


Table 2 Medline Search Strategy

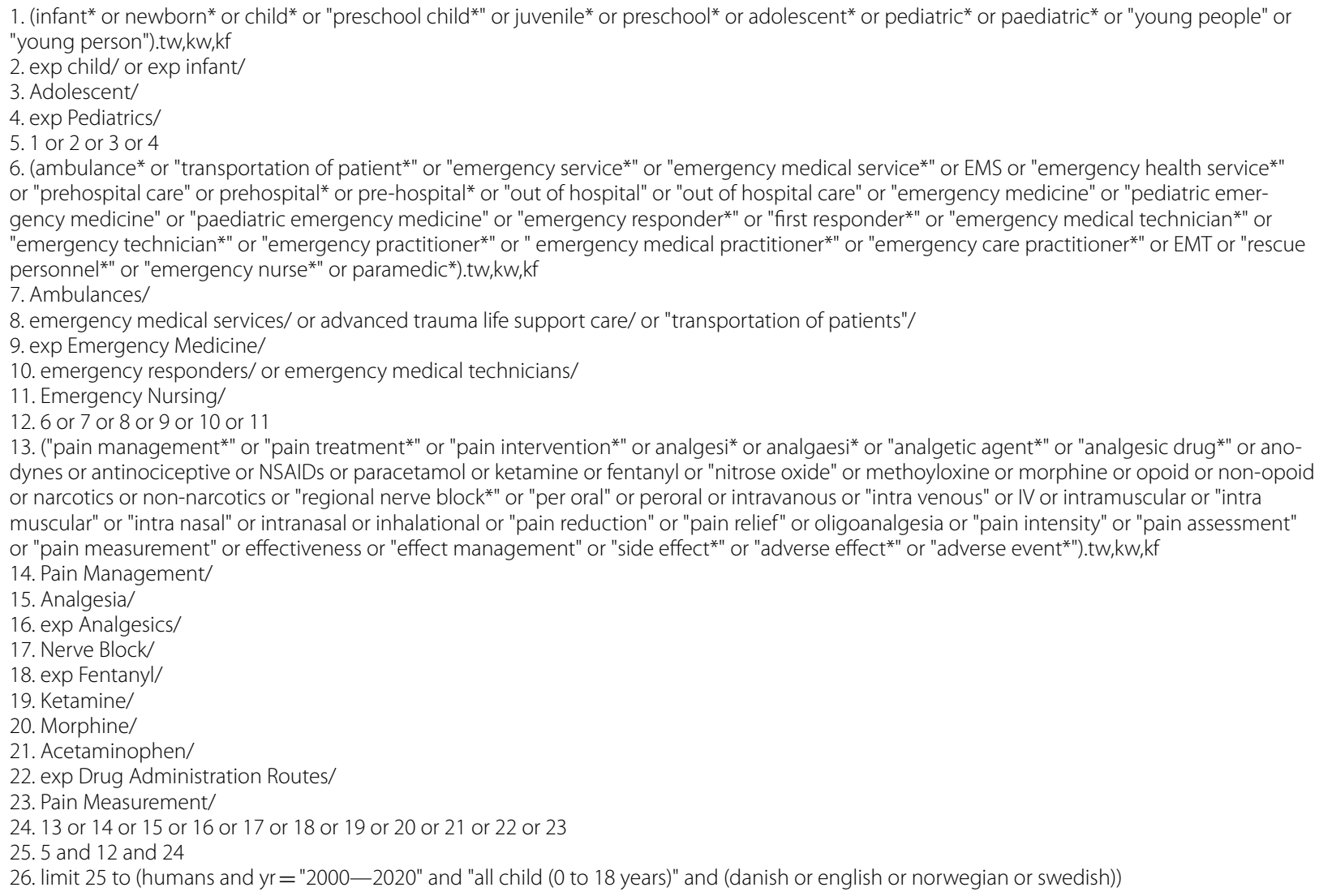

\section{Characteristics of the included studies}

According to the evidence evaluation worksheet developed by the International Liaison Committee on Resuscitation for therapeutic interventions, the current review found level three and four evidence only, despite using a broad search strategy. Among the eight included studies, six were cross-sectional studies while the remaining two were case series and cohort studies, respectively. All studies were conducted in high-income countries; five in Australia [30, 31, 33, 34, 36] and three in Europe [32, 35, 37]. The publication year ranges from 2006 to 2017. The studies involved a total of 71,674 study participants. Six studies examined the paediatrics age group only. The other two [32,34] included both adults and paediatrics (both had a separate report for the paediatric age group). The overall characteristics of the included studies are presented in Table 9.

\section{Types of drugs}

Six studies [30-33, 36, 37] examined the effectiveness of the reported analgesic drugs while two did not address this outcome [34, 35]. Furthermore, five studies [30, 32,
$34,35,37]$ assessed the safety of the drugs, while the other three focused solely on their effectiveness [31, 33, 36].

\section{Effectiveness and safety of intranasal Fentanyl as a single analgesic drug}

Five of the studies [31-33, 36, 37] evaluated fentanyl as a single analgesic drug in children. Jennings describes fentanyl administration via both intranasal and IV routes (the results were not specified separately for each route of administration) [33], while the other studies addressed fentanyl administration via the intranasal route only. Each of the five studies identified fentanyl as an effective analgesic drug. Of these, two studies [32, 37] found that Intranasal Fentanyl (INF) given at a dose of $50 \mu \mathrm{g}$ as a single dose (mean cumulative dose of $114 \mu \mathrm{g}$ ) [32] and a total of $1.5 \mu \mathrm{g} / \mathrm{kg}$ (initial mean (SD) dose of $50( \pm 10) \mu \mathrm{g}$ ) [37] had no serious adverse events. The most common minor adverse events reported due to the administration of fentanyl are presented in Table 9. The dosage and any adverse events of fentanyl were not described in the other three studies [31, 33, 36] 
Table 3 Embase Search Strategy

1. (infant* or newborn* or child* or "preschool child*" or juvenile* or preschool* or adolescent* or pediatric* or paediatric* or "young people" or "young person").tw,kw

2. child/ or juvenile/ or infant/ or preschool child/ or school child/ or toddler/

3. adolescent/

4. newborn/

5. pediatrics/ or pediatric emergency medicine/

6. 1 or 2 or 3 or 4 or 5

7. (ambulance* or "transportation of patient*" or "emergency service*" or "emergency medical service*" or EMS or "emergency health service*" or "prehospital care" or prehospital* or pre-hospital* or "out of hospital" or "out of hospital care" or "emergency medicine" or "pediatric emergency medicine" or "paediatric emergency medicine" or "emergency responder*" or "first responder*" or "emergency medical technician*" or "emergency technician*" or "emergency practitioner*" or " emergency medical practitioner*" or "emergency care practitioner*" or EMT or "rescue personnel*" or "emergency nurse*" or paramedic*).tw,kw

8. ambulance/

9. emergency health service/

10. exp rescue personnel/

11. emergency nursing/

12. emergency medicine/ or pediatric emergency medicine/

13.7 or 8 or 9 or 10 or 11 or 12

14. ("pain management*" or "pain treatment*" or "pain intervention*" or analgesi* or analgaesi* or "analgetic agent*" or "analgesic drug*" or anodynes or antinociceptive or NSAIDs or paracetamol or ketamine or fentanyl or "nitrose oxide" or methoyloxine or morphine or opoid or non-opoid or narcotics or non-narcotics or "regional nerve block*" or "per oral" or peroral or intravanous or "intra venous" or IV or intramuscular or "intra muscular" or "intra nasal" or intranasal or inhalational or "pain reduction" or "pain relief" or oligoanalgesia or "pain intensity" or "pain assessment" or "pain measurement" or effectiveness or "effect management" or "side effect*" or "adverse effect*" or "adverse event*").tw,kw

15. analgesia/

16. analgesic agent/

17. nerve block/

18. fentanyl/

19. ketamine/

20. morphine/

21. paracetamol/

22. exp drug administration route/

23. pain measurement/ or numeric rating scale/

24. pain assessment

25.14 or 15 or 16 or 17 or 18 or 19 or 20 or 21 or 22 or 23 or 24

26.6 and 13 and 25

27. limit 26 to (human and (danish or english or norwegian or swedish) and $y r=" 2000-2020 "$ and (infant or child or preschool child $<1$ to

6 years $>$ or school child $<7$ to 12 years $>$ or adolescent $<13$ to 17 years $>$ ))

\section{Effectiveness and safety of Morphine as a single analgesic drug}

Among the eight included studies, three investigated the use of morphine [31, 33, 36], all of which described the effectiveness of morphine administration in pain reduction. None of these studies stated the drug dosages or any adverse events.

In the study by Lord [31], 65\% of morphine administration was via the IV route while other routes of administration were not described. The other two studies [33, 36] examined morphine administration via the IV route only. These two studies also compared the effect of morphine and fentanyl on pain reduction, and both found that morphine had an equivalent effect to fentanyl in pain reduction.

\section{Effectiveness and safety of inhalational Methoxyflurane as a single analgesic drug}

A total of five studies [30, 31, 33, 34, 36] addressed the use of inhalational Methoxyflurane (IHM). Of these, three studies [31, 33, 36] examined the effectiveness of the drug, while the other two $[30,34]$ focused on adverse events related to IHM.

A case series conducted over a time span of eight months in Australia identified a mean pain reduction from the initial pain score of 7.9-3.2 after $10 \mathrm{~min}$ of IHM administration [30]. In this study, about $91.4 \%$ of children received a single dose of $3 \mathrm{ml}$ IHM while the others received two doses. In addition, only ten (9.5\%) patients received additional IV morphine after the IHM administration had commenced. No serious adverse events had been caused by the IHM administration. About 33.3\% and $8 \%$ of children under and over five years, respectively, developed deep sedation after receiving IHM. None of these deeply sedated patients had received additional IV morphine. They had immediately regained full consciousness within minutes as methoxyflurane administration was discontinued, and no further measures were needed. In addition, no renal impairment was reported from methoxyflurane administration. The most common minor adverse events found in this study are presented in Table 9. 
Table 4 PubMed Search Strategy

\#1. infant*[Title/Abstract] OR newborn*[Title/Abstract] OR child*[Title/Abstract] OR "preschool child*"[Title/Abstract] OR juvenile*[Title/Abstract] OR preschool*[Title/Abstract] OR adolescent*[Title/Abstract] OR pediatric*[Title/Abstract] OR paediatric*[Title/Abstract] OR "young people"[Title/ Abstract] OR "young person"[Title/Abstract]

\#2. "Child"[Mesh] OR "Infant"[Mesh] OR "Adolescent"[Mesh] OR "Pediatrics"[Mesh]

\#3. \#1 OR \#2

\#4. ambulance*[Title/Abstract] OR "transportation of patient*"[Title/Abstract] OR "emergency service*"[Title/Abstract] OR "emergency medical service*"[Title/Abstract] OR EMS[Title/Abstract] OR "emergency health service*"[Title/Abstract] OR "prehospital care"[Title/Abstract] OR prehospital*[Title/Abstract] OR pre-hospital*[Title/Abstract] OR "out of hospital"[Title/Abstract] OR "out of hospital care"[Title/Abstract] OR "emergency medicine"[Title/Abstract] OR "pediatric emergency medicine"[Title/Abstract] OR "paediatric emergency medicine"[Title/Abstract] OR "emergency responder*"[Title/Abstract] OR "first responder*"[Title/Abstract] OR "emergency medical technician*"[Title/Abstract] OR "emergency technician*"[Title/Abstract] OR "emergency practitioner*"[Title/Abstract] OR " emergency medical practitioner*"[Title/Abstract] OR "emergency care practitioner*"[Title/Abstract] OR EMT[Title/Abstract] OR "rescue personnel*"[Title/Abstract] OR "emergency nurse*"[Title/Abstract] OR paramedic*[Title/Abstract]

\#5. "Emergency Medical Services"[Mesh] OR "Emergency Medicine"[Mesh] OR "Emergency Medical Technicians"[Mesh] \#6. \#4 OR \#5

\#7. "pain management*"[Title/Abstract] OR "pain treatment*"[Title/Abstract] OR "pain intervention*"[Title/Abstract] OR analgesi*[Title/ Abstract] OR analgaesi*[Title/Abstract] OR "analgetic agent*"[Title/Abstract] OR "analgesic drug*"[Title/Abstract] OR anodynes[Title/Abstract] OR antinociceptive[Title/Abstract] OR NSAIDs[Title/Abstract] OR paracetamol[Title/Abstract] OR ketamine[Title/Abstract] OR fentanyl[Title/ Abstract] OR "nitrose oxide"[Title/Abstract] OR methoyloxine[Title/Abstract] OR morphine[Title/Abstract] OR opoid[Title/Abstract] OR nonopoid[Title/Abstract] OR narcotics[Title/Abstract] OR non-narcotics[Title/Abstract] OR "regional nerve block*"[Title/Abstract] OR "per oral"[Title/ Abstract] OR peroral[Title/Abstract] OR intravanous[Title/Abstract] OR "intra venous"[Title/Abstract] OR IV[Title/Abstract] OR intramuscular[Title/ Abstract] OR "intra muscular"[Title/Abstract] OR "intra nasal"[Title/Abstract] OR intranasal[Title/Abstract] OR inhalational[Title/Abstract] OR "pain reduction"[Title/Abstract] OR "pain relief"[Title/Abstract] OR oligoanalgesia[Title/Abstract] OR "pain intensity"[Title/Abstract] OR "pain assessment"[Title/Abstract] OR "pain measurement"[Title/Abstract] OR effectiveness[Title/Abstract] OR "effect management"[Title/Abstract] OR "side effect*"[Title/Abstract] OR "adverse effect*"[Title/Abstract] OR "adverse event*"[Title/Abstract]

\#8. "Pain Management"[Mesh] OR "Analgesia"[Mesh] OR "Analgesics"[Mesh] OR "Nerve Block"[Mesh] OR "Fentanyl"[Mesh] OR "Ketamine"[Mesh] OR "Morphine"[Mesh] OR "Acetaminophen"[Mesh] OR "Drug Administration Routes"[Mesh] OR "Pain Measurement"[Mesh]

\#9. \#7 OR \#8

\#10. \#3 AND \#6 AND \#9

\#11. \#3 AND \#6 AND \#9 Filters: Humans, Danish, English, Norwegian, Swedish, Child: birth-18 years, from 2000—2020

One cohort study [34] conducted a separate analysis of 594 patients under the age of 12 years who received methoxyflurane, all of whom received a single IHM dose of $3 \mathrm{ml}(0.3 \%)$. This study did not address the effectiveness of the drug. However, it found no observed increased risk of disease occurrence following methoxyflurane administration when compared to a similar group of patients who did not receive methoxyflurane. The investigated outcome variables among the exposed and control group were the presence of ischemic heart disease, diabetes, renal disease, cancer and hepatic diseases.

The other three retrospective cross-sectional studies conveyed the effectiveness of methoxyflurane in pain reduction [31, 33, 36]. In addition, Bendall found that IHM had less analgesic effect when compared to morphine, fentanyl and combined agents (AOR 0.52; 95\% CI 0.36-0.74) [36]. In contrast, another study [33] reported that methoxyflurane had the greatest odds of achieving clinically meaningful pain reduction when compared to morphine and fentanyl (AOR 5.3; 95\% CI 4.8-5.9). The drug doses and/or any adverse events were not described in any of the three retrospective cross-sectional studies.

\section{Effectiveness and safety of Ketamine as a single analgesic drug}

Only one retrospective database review examined the use of ketamine in children below the age of 16 [35]. The mean administered drug dose was $1.0 \mathrm{mg} / \mathrm{kg}$ (ranges from 0.1 to $5.8 \mathrm{mg} / \mathrm{kg}$ ). The route of administration was IV (86\%) and intramuscular (IM) (14\%). A majority (68\%) of patients also received a mean dose of $0.1 \mathrm{mg} / \mathrm{kg}$ midazolam as a co-drug. The study did not look at the effectiveness of the drug. No deaths or any implementation of basic airway manoeuvres had occurred due to ketamine administration. In all, only one adverse event had been recorded, which was desaturation (desaturation $<4 \%$ ) in only four (2.4\%) patients. Furthermore, the study did not find desaturation in children younger than three years of age after receiving an analgesic dose of ketamine.

\section{Effectiveness and safety of combination analgesic drugs}

Three studies examined the effectiveness of combination analgesic drugs [31, 36, 37]. All three identified the effectiveness of the use of combination drugs. None of these studies examined adverse events from the use of combination drugs.

The first study reported that intranasal fentanyl in combination with paracetamol \pm ibuprofen \pm inhaled nitrous oxide had a $79 \%$ effectiveness in pain reduction [37]. The second study showed that a combination of the three drugs (morphine, fentanyl and methoxyflurane) had a median pain score change of 4 (IQR 3-6) [31]. The third study identified that the use of a combination of more than one drug from the three analgesics (morphine, 
Table 5 CINAHL Search Strategy

S1. TI (infant* or newborn* or child* or "preschool child*" or juvenile* or preschool* or adolescent* or pediatric* or paediatric* or "young people" or "young person") OR AB ( infant* or newborn* or child* or "preschool child*" or juvenile* or preschool* or adolescent* or pediatric* or paediatric* or "young people" or "young person")

S2. (MH "Child + ") OR (MH "Infant+")

S3. (MH "Adolescence")

S4. (MH "Pediatrics")

S5. S1 OR S2 OR S3 OR S4

S6. TI ( ambulance* or "transportation of patient*" or "emergency service*" or "emergency medical service*" or EMS or "emergency health service*" or "prehospital care" or prehospital* or pre-hospital* or "out of hospital" or "out of hospital care" or "emergency medicine" or "pediatric emergency medicine" or "paediatric emergency medicine" or "emergency responder*" or "first responder*" or "emergency medical technician*" or "emergency technician*" or "emergency practitioner*" or " emergency medical practitioner*" or "emergency care practitioner*" or EMT or "rescue personnel*" or "emergency nurse*" or paramedic*) OR AB ( ambulance* or "transportation of patient*" or "emergency service*" or "emergency medical service*" or EMS or "emergency health service*" or "prehospital care" or prehospital* or pre-hospital* or "out of hospital" or "out of hospital care" or "emergency medicine" or "pediatric emergency medicine" or "paediatric emergency medicine" or "emergency responder*" or "first responder*" or "emergency medical technician*" or "emergency technician*" or "emergency practitioner*" or " emergency medical practitioner*" or "emergency care practitioner*" or EMT or "rescue personnel*" or "emergency nurse*" or paramedic*)

S7. (MH "Prehospital Care")

S8. (MH "Emergency Medical Services") OR (MH "Transportation of Patients")

59. (MH "Ambulances")

S10. (MH "Emergency Medicine")

S11. (MH "Emergency Medical Technicians")

S12. S6 OR S7 OR S8 OR S9 OR S10 OR S11

S13. TI ( "pain management*" or "pain treatment*" or "pain intervention*" or analgesi* or analgaesi* or "analgetic agent*" or "analgesic drug*" or anodynes or antinociceptive or NSAIDs or paracetamol or ketamine or fentanyl or "nitrose oxide" or methoyloxine or morphine or opoid or non-opoid or narcotics or non-narcotics or "regional nerve block*" or "per oral" or peroral or intravanous or "intra venous" or IV or intramuscular or "intra muscular" or "intra nasal" or intranasal or inhalational or "pain reduction" or "pain relief" or oligoanalgesia or "pain intensity" or "pain assessment" or "pain measurement" or effectiveness or "effect management" or "side effect*" or "adverse effect*" or "adverse event*") OR AB ( "pain management*" or "pain treatment*" or "pain intervention*" or analgesi* or analgaesi* or "analgetic agent*" or "analgesic drug*" or anodynes or antinociceptive or NSAIDs or paracetamol or ketamine or fentanyl or "nitrose oxide" or methoyloxine or morphine or opoid or non-opoid or narcotics or non-narcotics or "regional nerve block*" or "per oral" or peroral or intravanous or "intra venous" or IV or intramuscular or "intra muscular" or "intra nasal" or intranasal or inhalational or "pain reduction" or "pain relief" or oligoanalgesia or "pain intensity" or "pain assessment" or "pain measurement" or effectiveness or "effect management" or "side effect*" or "adverse effect*" or "adverse event*")

S14. (MH "Pain Management")

S15. (MH "Analgesia")

S16. (MH "Analgesics+")

S17. (MH "Nerve Block")

S18. (MH "Acetaminophen")

S19. (MH "Drug Administration Routes + ")

S20. (MH "Pain Measurement")

S21. S13 OR S14 OR S15 OR S16 OR S17 OR S18 OR S19 OR S20

S22. S5 AND S12 AND S21

S23. S5 AND S12 AND S21 Limiters—Age Groups: Infant, Newborn: birth-1 month, Infant: 1-23 months, Child, Preschool: 2-5 years, Child:

6-12 years, Adolescent: 13-18 years, All Infant, All Child; Published Date: 20,000,101-20,201,231; Human; Language: Danish, English, Norwegian, Swedish

fentanyl and methoxyflurane) had a statistically significant higher median pain score difference (median pain score difference of 6 (IQR 4-7)) compared to use of the drugs independently. However, there was no statistical evidence suggesting that combination drugs were more effective than morphine or fentanyl alone after controlling for factors such as age and gender [36].

\section{Risk of bias}

The JBI critical appraisal checklist was used to assess the risk of bias. The JBI checklists for analytical cross-sectional, prevalence, case series and cohort study designs were used. Nine questions were assessed using the prevalence study design checklist, eight in the analytical, ten in the case series and eleven in the cohort study design. The findings of the assessment are provided in Table 10.

\section{Discussion}

The present systematic review on the effectiveness and safety of analgesic drugs used in prehospital pain relief in children identified a total of eight articles. Most of these articles concerned analgesic drugs such as fentanyl (intranasal/IV), morphine (IV), methoxyflurane (inhalational) and ketamine (IV/IM). The studies examine the effects of fentanyl (intranasal/IV), morphine (IV) and methoxyflurane (inhalational), and all of the analgesic drugs were found to be effective. Adverse events of intranasal fentanyl, inhalational methoxyflurane and IV/IM ketamine 
Table 6 Cochrane Library Search Strategy

\#1. (infant* or newborn* or child* or "preschool child*" or juvenile* or preschool* or adolescent* or pediatric* or paediatric* or "young people" or "young person"):ti,ab,kw

\#2. MeSH descriptor: [Child] explode all trees

\#3. MeSH descriptor: [Adolescent] explode all trees

\#4. MeSH descriptor: [Infant] explode all trees

\#5. MeSH descriptor: [Pediatrics] explode all trees

\#6. \#1 or \#2 or \#3 or \#4 or \#5

\#7. (ambulance* or "transportation of patient*" or "emergency service*" or "emergency medical service*" or EMS or "emergency health service*" or "prehospital care" or prehospital* or pre-hospital* or "out of hospital" or "out of hospital care" or "emergency medicine" or "pediatric emergency medicine" or "paediatric emergency medicine" or "emergency responder*" or "first responder*" or "emergency medical technician*" or "emergency technician*" or "emergency practitioner*" or " emergency medical practitioner*" or "emergency care practitioner*" or EMT or "rescue personnel*" or "emergency nurse*" or paramedic*):ti,ab,kw

\#8. MeSH descriptor: [Emergency Medical Services] explode all trees

\#9. MeSH descriptor: [Ambulances] in all MeSH products

$\# 10$. MeSH descriptor: [Emergency Medicine] explode all trees

\#11. MeSH descriptor: [Emergency Medical Technicians] explode all trees

\#12. MeSH descriptor: [Emergency Nursing] explode all trees

\#13. \#7 or \#8 or \#9 or \#10 or \#11 or \#12

\#14. ("pain management*" or "pain treatment*" or "pain intervention*" or analgesi* or analgaesi* or "analgetic agent*" or "analgesic drug*" or anodynes or antinociceptive or NSAIDs or paracetamol or ketamine or fentanyl or "nitrose oxide" or methoyloxine or morphine or opoid or non-opoid or narcotics or non-narcotics or "regional nerve block*" or "per oral" or peroral or intravanous or "intra venous" or IV or intramuscular or "intra muscular" or "intra nasal" or intranasal or inhalational or "pain reduction" or "pain relief" or oligoanalgesia or "pain intensity" or "pain assessment" or "pain measurement" or effectiveness or "effect management" or "side effect*" or "adverse effect*" or "adverse event*"):ti,ab,kw \#15. MeSH descriptor: [Pain Management] explode all trees

\#16. MeSH descriptor: [Analgesia] explode all trees

\#17. MeSH descriptor: [Analgesics] explode all trees

\#18. MeSH descriptor: [Nerve Block] explode all trees

\#19. MeSH descriptor: [Fentanyl] explode all trees

\#20. MeSH descriptor: [Ketamine] explode all trees

\#21. MeSH descriptor: [Morphine] in all MeSH products

\#22. MeSH descriptor: [Acetaminophen] explode all trees

\#23. MeSH descriptor: [Drug Administration Routes] explode all trees

\#24. MeSH descriptor: [Pain Measurement] explode all trees

\#25. \#14 or \#15 or \#16 or \#17 or \#18 or \#19 or \#20 or \#21 or \#22 or \#23 or \#24

$\# 26 . \# 6$ and \#13 and \#25

\#27. \#6 and \#13 and \#25 with Cochrane Library publication date from Jan 2000 to Dec 2020

Table 7 Epistemonikos Search Strategy

(title:(infant* OR newborn* OR child* OR "preschool child*" OR juvenile* OR preschool* OR adolescent* OR pediatric* OR paediatric* OR "young people" OR "young person") OR abstract:(infant* OR newborn* OR child* OR "preschool child*" OR juvenile* OR preschool* OR adolescent* OR pediatric* OR paediatric* OR "young people" OR "young person")) AND (title:(ambulance* OR "transportation of patient*" OR "emergency service*" OR "emergency medical service*" OR EMS OR "emergency health service*" OR "prehospital care" OR prehospital* OR pre-hospital* OR "out of hospital" OR "out of hospital care" OR "emergency medicine" OR "pediatric emergency medicine" OR "paediatric emergency medicine" OR "emergency responder*" OR "first responder*" OR "emergency medical technician*" OR "emergency technician*" OR "emergency practitioner*" OR " emergency medical practitioner*" OR "emergency care practitioner*" OR EMT OR "rescue personnel*" OR "emergency nurse*" OR paramedic*) OR abstract:(ambulance* OR "transportation of patient*" OR "emergency service*" OR "emergency medical service*" OR EMS OR "emergency health service*" OR "prehospital care" OR prehospital* OR pre-hospital* OR "out of hospital" OR "out of hospital care" OR "emergency medicine" OR "pediatric emergency medicine" OR "paediatric emergency medicine" OR "emergency responder*" OR "first responder*" OR "emergency medical technician*" OR "emergency technician*" OR "emergency practitioner*" OR " emergency medical practitioner*" OR "emergency care practitioner*" OR EMT OR "rescue personnel*" OR "emergency nurse*" OR paramedic*)) AND (title:("pain management*" OR "pain treatment*" OR "pain intervention*" OR analgesi* OR analgaesi* OR "analgetic agent*" OR "analgesic drug*" OR anodynes OR antinociceptive OR NSAIDs OR paracetamol OR ketamine OR fentanyl OR "nitrose oxide" OR methoyloxine OR morphine OR opoid OR non-opoid OR narcotics OR non-narcotics OR "regional nerve block*" OR "per oral" OR peroral OR intravanous OR "intra venous" OR IV OR intramuscular OR "intra muscular" OR "intra nasal" OR intranasal OR inhalational OR "pain reduction" OR "pain relief" OR oligoanalgesia OR "pain intensity" OR "pain assessment" OR "pain measurement" OR effectiveness OR "effect management" OR "side effect*" OR "adverse effect*" OR "adverse event*") OR abstract:("pain management*" OR "pain treatment*" OR "pain intervention*" OR analgesi* OR analgaesi* OR "analgetic agent*" OR "analgesic drug*" OR anodynes OR antinociceptive OR NSAIDs OR paracetamol OR ketamine OR fentanyl OR "nitrose oxide" OR methoyloxine OR morphine OR opoid OR non-opoid OR narcotics OR non-narcotics OR "regional nerve block*" OR "per oral" OR peroral OR intravanous OR "intra venous" OR IV OR intramuscular OR "intra muscular" OR "intra nasal" OR intranasal OR inhalational OR "pain reduction" OR "pain relief" OR oligoanalgesia OR "pain intensity" OR "pain assessment" OR "pain measurement" OR effectiveness OR "effect management" OR "side effect*" OR "adverse effect*" OR "adverse event*"))limit 2000-2020 
Table 8 Levels of Evidence (LOE) for Studies of Therapeutic Interventions

\begin{tabular}{ll}
\hline LOE 1 & Randomised Controlled Trials (or meta-analyses of RCTs) \\
\hline LOE 2 & Studies using concurrent controls without true randomisation (e.g. "pseudo"-randomised) \\
LOE 3 & Studies using retrospective controls \\
LOE 4 & Studies without a control group (e.g. case series) \\
LOE 5 & Studies not directly related to the specific patient/population (e.g. different patient/ \\
& population, animal models, mechanical models, etc.) \\
\hline
\end{tabular}

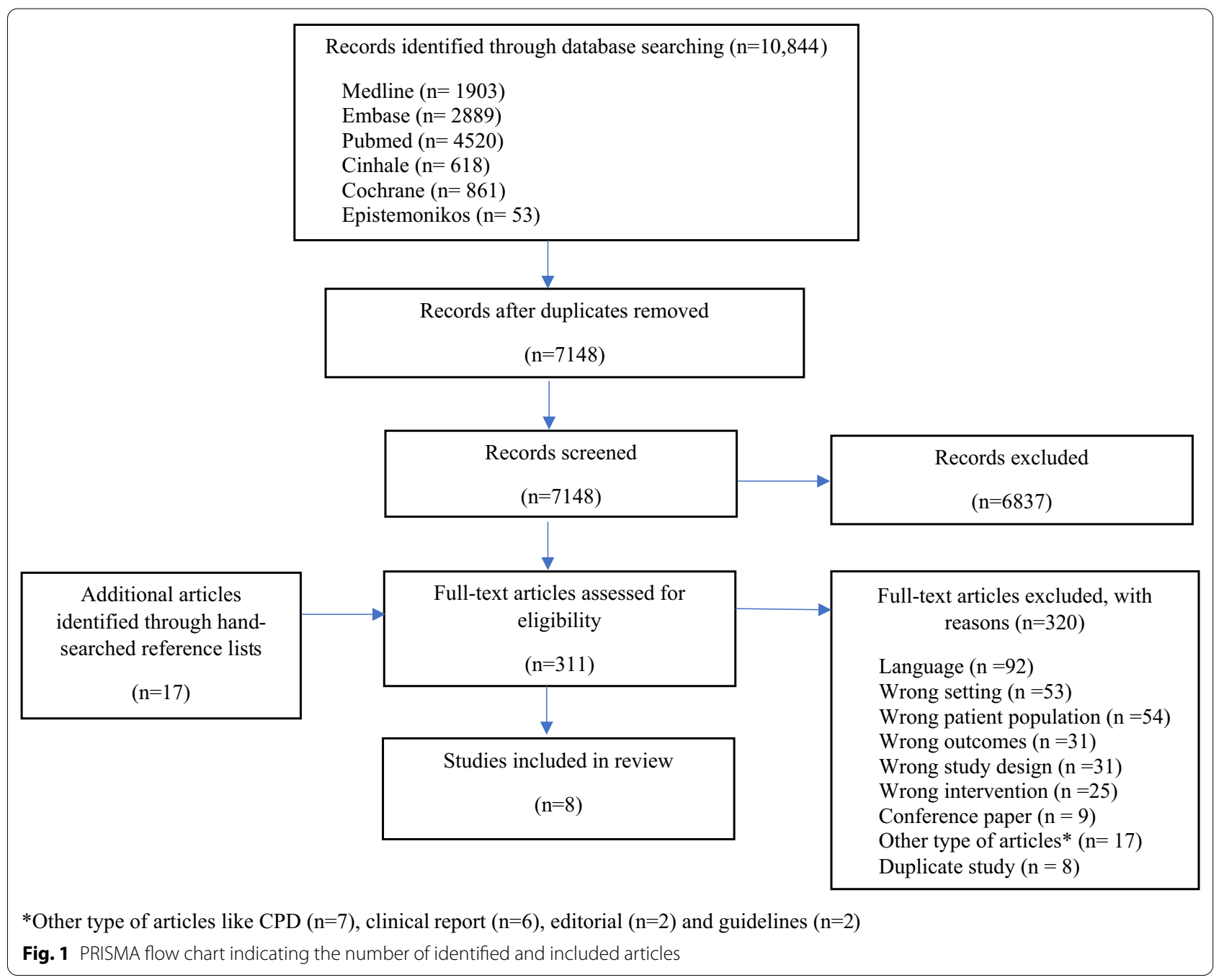

were also described. However, none of these drugs were found to have serious adverse events.

The Italian Intersociety Recommendations on pain management in emergency settings stated that 'the ideal prehospital analgesic should be easy to use, safe, effective, and have a predictable dose-response relationship with rapid onset and a short duration of action' [38]. Our results show that both intranasal fentanyl and inhalational methoxyflurane are effective and safe to administer both as single drugs and/or in combination with other examined analgesic drugs. Similarly, several studies conducted in different acute care settings support that intranasal fentanyl and inhalational methoxyflurane are effective analgesic drugs with no serious adverse events [19, 39-43]. It has been suggested that these drugs are easy to administer and have a rapid onset and short duration of action [42, 44, 45]. Moreover, the present review found that fentanyl has an equivalent effect to morphine. 


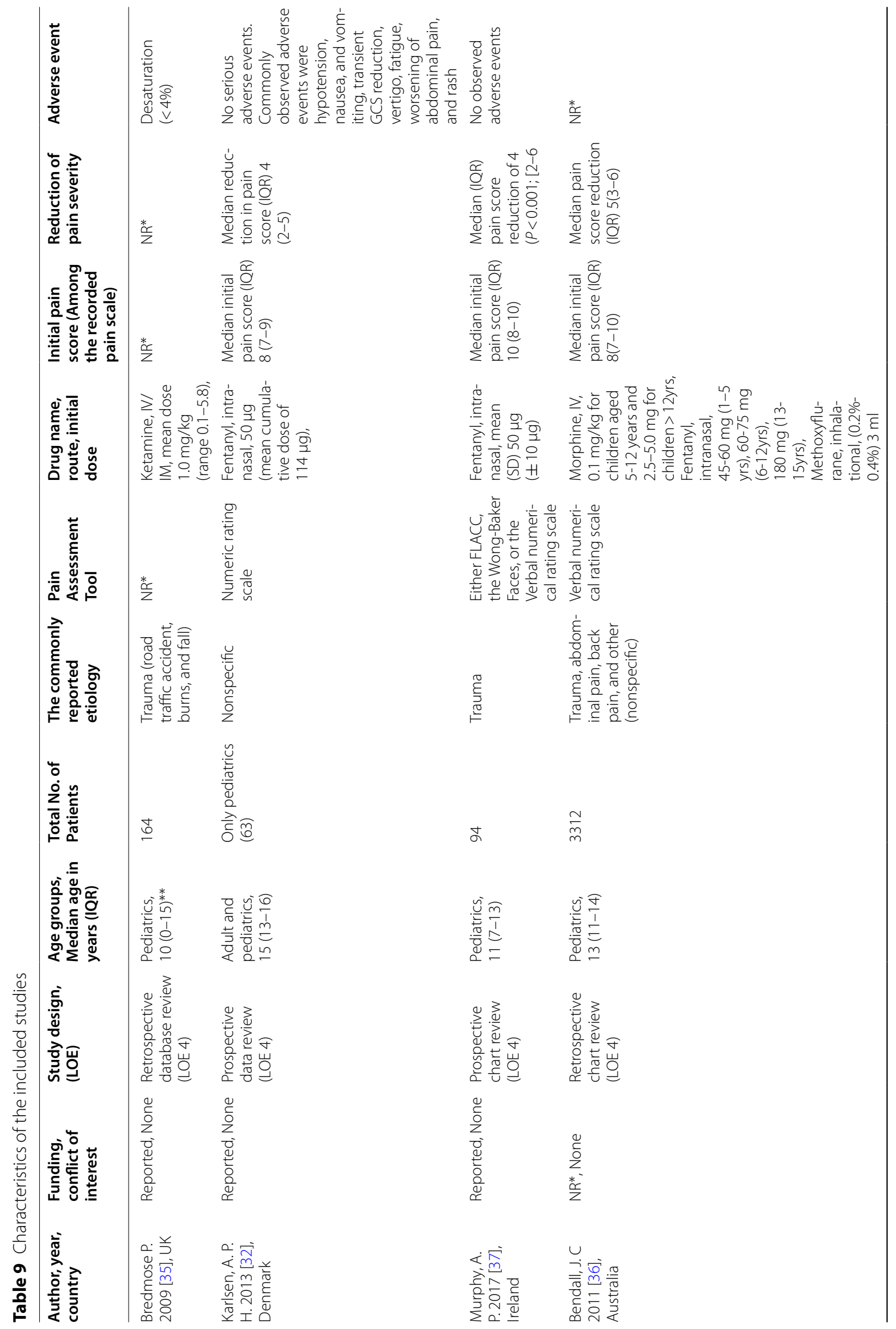




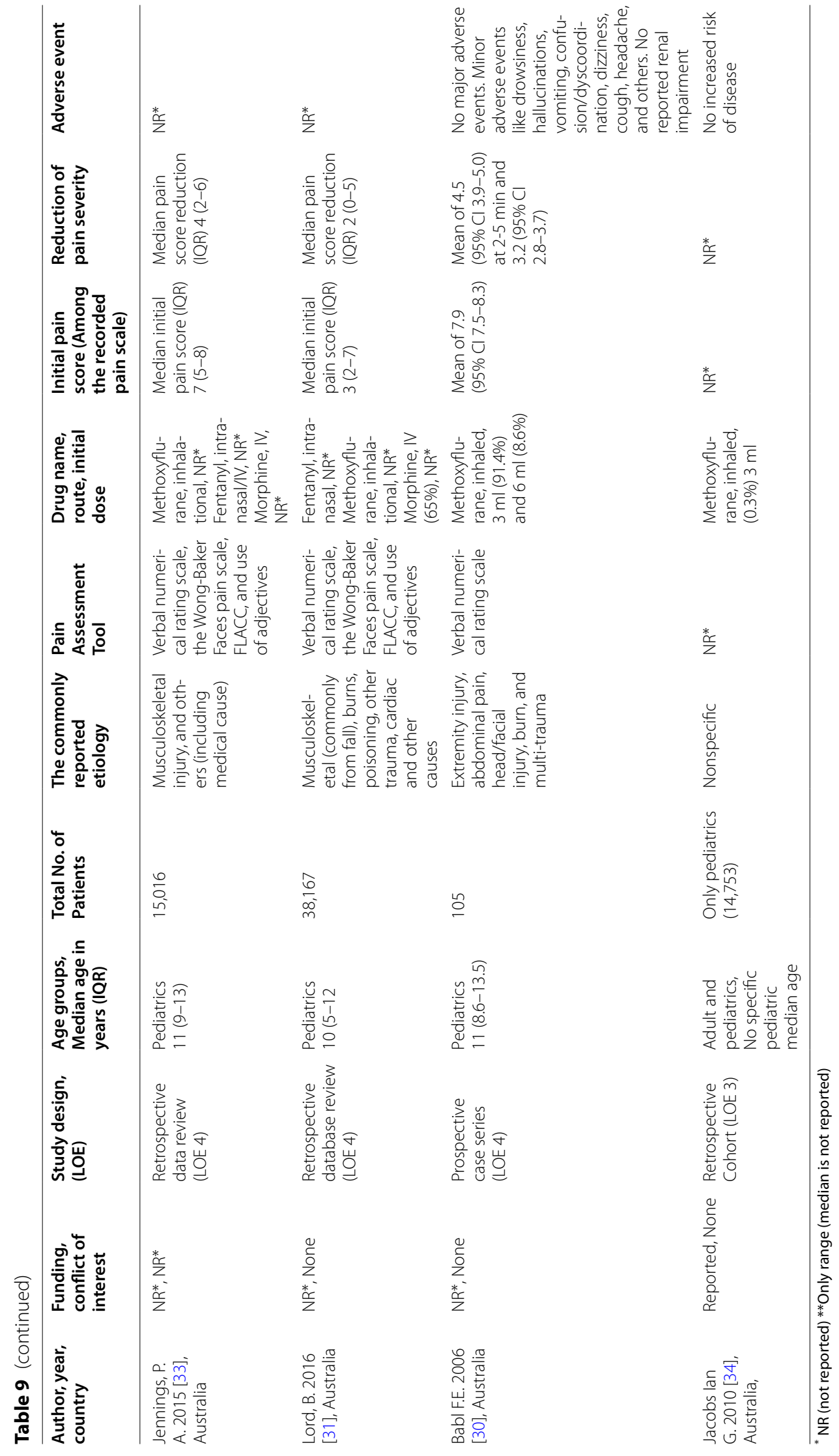




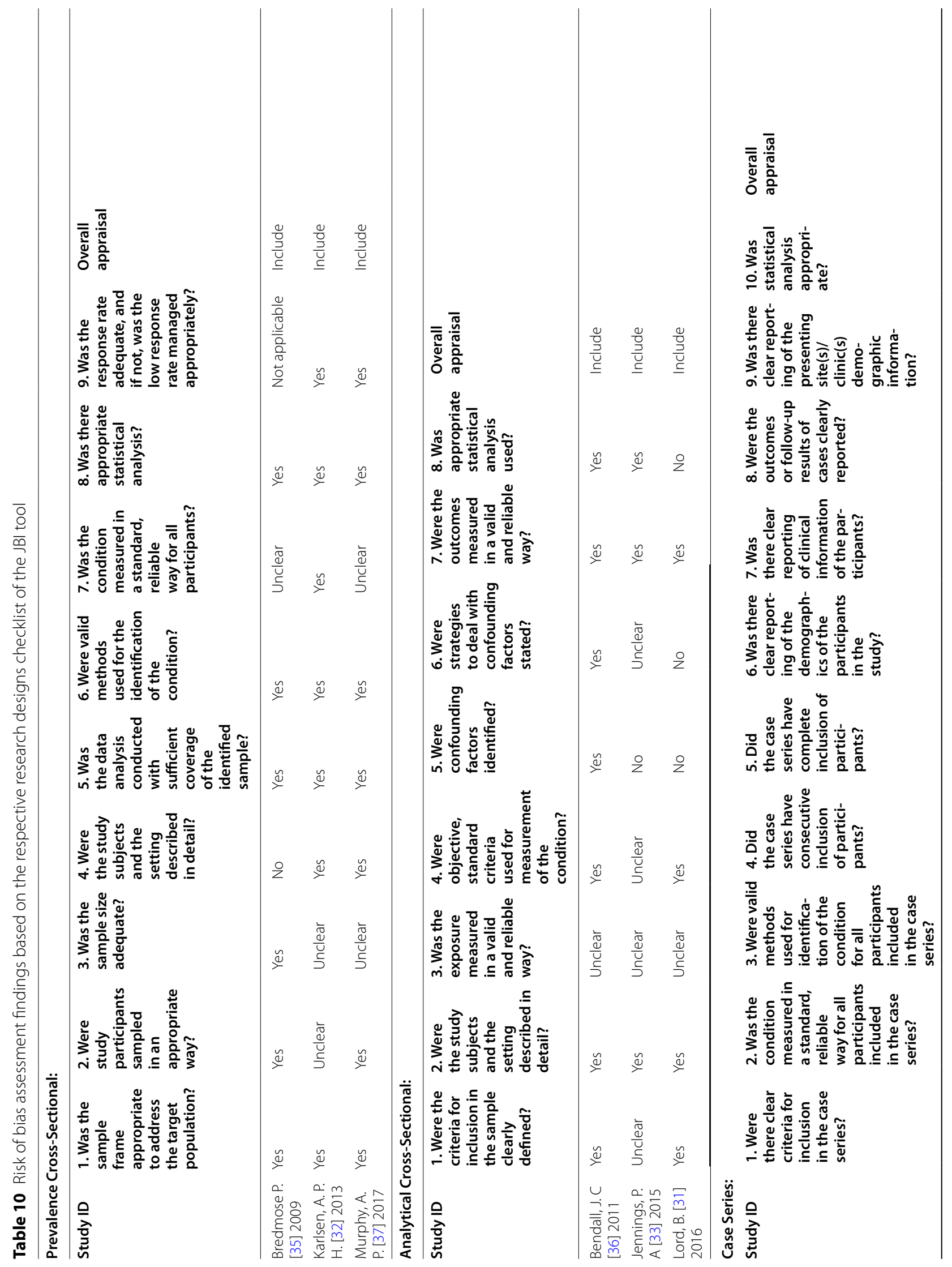




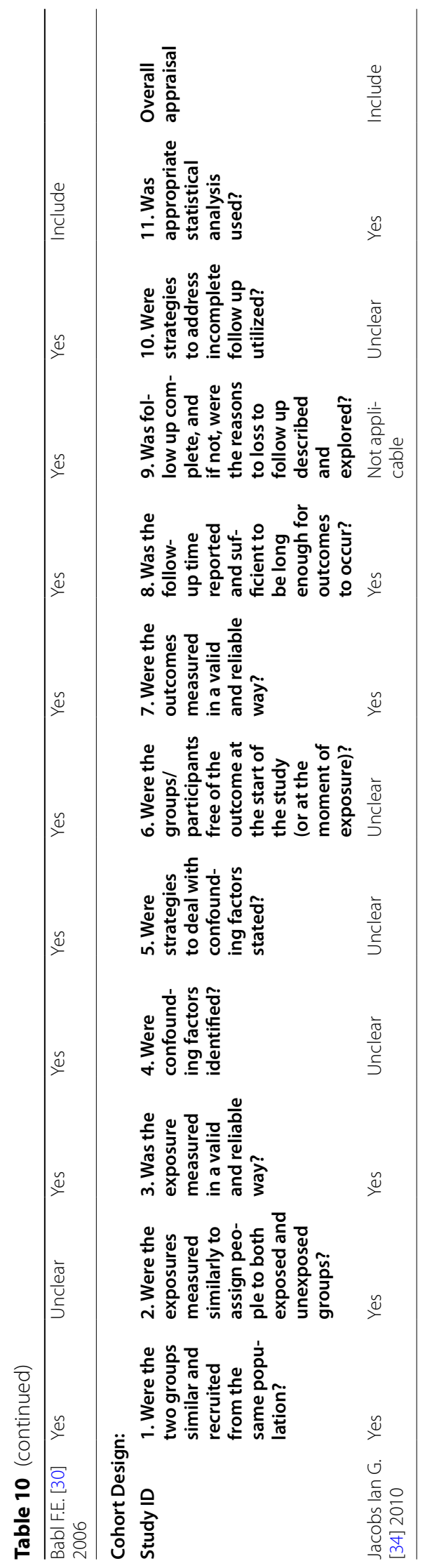


A randomised control trial conducted in Australian emergency departments also found similar results [39]. Therefore, intranasal fentanyl and inhalational methoxyflurane seem to be the drugs of choice due to their ease of administration, rapid onset, short duration of action, effect and safety profile for children's pain relief in prehospital settings. However, it can be difficult to administer inhalational methoxyflurane in non-cooperative children and in cases involving facial trauma. Similarly, contraindications for nasal drug administrations could be a limitation to administering intranasal fentanyl.

Morphine (IV) was identified in the present systematic analysis as an effective analgesic drug, but the safety of the drug was not evaluated in any of the studies included in this review. This is supported by previous results, and IV morphine has traditionally been reported as the gold standard drug for acute pain relief in acute care settings $[40,46,47]$. Furthermore, the findings support that analgesic doses of ketamine (IV/IM) are safe to use in acute care settings [48-50]. Although we could not find any studies that address the effectiveness of ketamine for the purpose of this review, previous studies have identified ketamine as an effective analgesic drug in acute care settings [48-50]. However, difficulties concerning IV access, anxiety related to painful IM injection and prolonged prehospital time are obvious limitations in IV/IM morphine and ketamine administration in the prehospital pain management of children [13, 40, 41].

The current review did not identify any serious adverse events from the analgesic drugs included in the studies. However, previous studies have demonstrated that opioids (morphine and fentanyl) have adverse events such as respiratory depression, apnoea, sedation, bradycardia and gastrointestinal dysmotility [51,52]. There is also a fear of sedation, respiratory depression, renal and hepatic failure related to methoxyflurane use [53, 54]. In addition, Ketamine has dose-dependent adverse events such as sedation, hypoxia, laryngospasm, hypersalivation, nausea and vomiting $[55,56]$. Hence, to minimise the potential risk due to these adverse events, opioids, methoxyflurane and ketamine should always be administered with caution. Naloxone for an opioid antagonist and/or airway opening and ventilatory devices should always be ready on hand [57].

\section{Limitations of the included studies and the current review}

Most of the studies included in this review were based on retrospective chart reviews. Poor data recording, underreport biases and selection biases are the major challenges of such designs. The training level, scope, and expertise of the care providers could also vary accordingly. It is unclear whether such variations would affect the effectiveness and safety of the included drugs. It would be good to study this in the future research.

Our systematic review has some limitations. Firstly, the systematic literature searches were limited by publication year (the past 20 years from 2000 to 2020) and language (English, Danish, Norwegian or Swedish). This was due to the purpose of having current pharmacological prehospital pain management modalities and due to the authors' language capabilities and expenses. Secondly, the corresponding authors of four of the included studies [32, 34, $36,37]$ did not respond to requests for further information when contacted.

Except in combination with opioids, the authors were not able to find studies that examine the effectiveness and safety of any other common analgesic drugs such as acetaminophen and Non-Steroidal Anti-Inflammatory Drugs (NSAIDs), which could be given for mild to moderate pain $[43,51,52]$. We were also unable to find studies related to the effects and safety of prehospital use of nitrous oxide (Entonox) and nerve block drugs in children.

\section{Conclusions}

Our systematic review revealed that fentanyl (intranasal/ IV), morphine (IV), methoxyflurane (inhalational) and combination drugs are effective analgesic drugs for children in prehospital settings. No serious adverse events were reported in the administration of intranasal fentanyl, inhalational methoxyflurane or IV/IM ketamine. Intranasal fentanyl and inhalational methoxyflurane seem to be the preferred drugs for children in pre-hospital settings due to their ease of administration, similar effect and safety profile when compared to other analgesic drugs. However, caution must be shown in reaching this conclusion since the included studies' level of evidence (LOE) was level three and four only.

This systematic review found that there is a paucity of high-level evidence on children's prehospital pain management. Furthermore, all of the studies included were conducted solely from the perspectives of high-income countries. Well-designed comprehensive studies that include the context of low- and middle-income countries should also be conducted. In addition to single analgesic drugs, multimodal analgesia also needs further analysis in future studies of prehospital pain management in children.

\footnotetext{
Abbreviations

CINAHL: Cumulative Index of Nursing and Allied Health Literature; CPDs: Continuing Professional Developments; DIKU: Norwegian Agency for International Cooperation and Quality Enhancement in Higher Education; ED: Emergency Department; Embase: Excerpta Medica Database; FDA: Food and Drug Administration; IHM: Inhalational Methoxyflurane; IM: Intramuscular; INF: Intranasal Fentanyl; IV: Intravenous; JBI: Joanna Briggs Institute; LOE: Level of Evidence;
} 
Medline: Medical Literature Analysis and Retrieval System Online; MeSH: Medical Subject Headings; NORPART: Norwegian Partnership Programme for Global Academic Cooperation; NSAIDs: Non-steroidal Anti-inflammatory Drugs; PRISMA: Preferred Reporting Items for Systematic Reviews and Meta-analyses; PROSPERO: International Prospective Register of Systematic Review.

\section{Acknowledgements}

The authors would like to thank Associate Professor Hilde Tinderholt Myrhaug and University Librarian Malene Wøhlk Gundersen, both of whom work at OsloMet, for their methodological advice and valuable suggestions on the search strategy.

\section{Authors' contributions}

The search strategy was developed by the four authors (YA, TS, FH and KS) who are the subject specialists, and was peer-reviewed by another author $(\mathrm{MH})$ who is a research librarian. The last searches were rerun by the research librarian (MH). The study selection was conducted by the four authors (YA, TS, $\mathrm{FH}$ and KS), respectively. The risk of bias was assessed by FH, KS and YA. Data extraction was conducted by YA and TS. All authors were involved in the data synthesis. YA wrote the draft manuscript and TS, FH and KS revised it accordingly. All authors read and approved the final manuscript.

\section{Funding}

The first author has received full funding from Oslo Metropolitan University (Norwegian Agency for International Cooperation and Quality Enhancement in Higher Education (DIKU/NORPART) project 2018/10182), Faculty of Health Sciences.

\section{Availability of data and materials}

All data generated or analysed during this study are included in this published article.

\section{Declarations}

\section{Ethics approval and consent to participate}

Not applicable.

\section{Consent for publication}

Not applicable.

\section{Competing interests}

The authors declare that they have no competing interests.

\section{Author details}

'Department of Emergency and Critical Care Nursing, St. Paul's Hospital Millennium Medical College, Addis Ababa, Ethiopia. ${ }^{2}$ Bachelor Programme in Paramedics, Institute of Nursing and Health Promotion, Faculty of Health Science, Oslo Metropolitan University, Oslo, Norway. ${ }^{3}$ Edge Hill University, Ormskirk, UK. ${ }^{4}$ Library and Knowledge Services for NHS Ambulance Services in England, Bolton, UK.

Received: 6 August 2021 Accepted: 28 October 2021

Published online: 11 December 2021

\section{References}

1 KD Friesgaard 2018 Acute pain in the prehospital setting: a registerbased study of 41.241 patients Scandinavian J Trauma Resuscit Emergency Med 26153

2 JTurner 2013 What outcome measures should be developed for prehospital care? Results of a consensus event Prehospital Disaster Med 28 Supp. 1589589

3 R Pagnamenta JR Benger 2008 Factors influencing parent satisfaction in a children's emergency department: prospective questionnaire-based study Emerg Med J 257417

4 M Galinski 2010 Prevalence and management of acute pain in prehospital emergency medicine Prehospital Emerg Care 143334339
5 A Murphy 2016 A prevalence and management study of acute pain in children attending emergency departments by ambulance Prehosp Emerg Care 2015258

6 B Lord M Woollard 2011 The reliability of vital signs in estimating pain severity among adult patients treated by paramedics Emerg Med J 282 147

7 H Hennes MK Kim RG Pirrallo 2005 PREHOSPITAL PAIN MANAGEMENT Prehosp Emerg Care 913239

8 R Pilbery J Miles F Bell 2019 A service evaluation of paediatric pain management in an English ambulance service Bt Paramedic J 4237

9 N Watkins 2006 Paediatric prehospital analgesia in Auckland Emerg Med Austral 181516

10 B DeBruyne 2014 Emergency medical services provider comfort with pre-hospital analgesia administration to children Can J Emerg Med 16 S52 S53

11 DM Williams 2012 Barriers to and enablers for prehospital analgesia for pediatric patients Prehosp Emerg Care 164519526

12 KM Adelgais K Brown 2014 Pediatric prehospital pain management: impact of advocacy and research Clin Pediatric Emerg Med 1514958

13 SH Thomas S Shewakramani 2008 Prehospital trauma analgesia J Emerg Med 3514757

14 R Sinatra 2010 Causes and consequences of inadequate management of acute pain Pain Med 111218591871

15 WT Zempsky NL Schechter 2003 What's new in the management of pain in children Pediatrics Rev 2410337347

16 SJ Weisman B Bernstein NL Schechter 1998 Consequences of inadequate analgesia during painful procedures in children Arch Pediatrics Adolescent Med 1522147149

17 JT Pate 1996 Childhood medical experience and temperament as predictors of adult functioning in medical situations Children's Health Care 254 281298

18 C Park 2010 Prehospital analgesia: systematic review of evidence BMJ Milit Health 156 Suppl 4 S295 300

19 N Samuel IP Steiner I Shavit 2015 Prehospital pain management of injured children: a systematic review of current evidence Am J Emerg Med 333451454

20 GA Whitley R Pilbery 2019 Pre-hospital intranasal analgesia for children suffering pain: a rapid evidence review Bt Paramedic J 4324

21 M Gausche-Hill 2014 An evidence-based guideline for prehospital analgesia in trauma Prehosp Emerg Care 18 sup 12534

22 MJ Page 2021 PRISMA 2020 explanation and elaboration: updated guidance and exemplars for reporting systematic reviews BMJ 372160

23 Fredrik Hetmann TS, Kacper Sumera, Matt Holland, Yonas Abebe Tessema. Pediatric prehospital pain management; a systematic review. 2019 [cited 2021 May 11]; Available from: https://www.crd.york.ac.uk/prospero/displ ay_record.php?!D=CRD42019126699.

24 B Bulloch M Tenenbein 2002 Assessment of clinically significant changes in acute pain in children Acad Emerg Med 93199202

25 (FDA), T.U.S.f.a.d.a. What is a Serious Adverse Event? 2016 [cited 2021 June 26]; Available from: https://www.fda.gov/safety/reporting-serious-probl ems-fda/what-serious-adverse-event.

26 KP Grichnik FM Ferrante 1991 The difference between acute and chronic pain Mt Sinai J Med 58321720

27 Covidence. Create and publish a data extraction template 2021 February 3, 2021 [cited 2021 May 28]; Available from: https://support.covidence. org/help/create-and-publish-a-data-extraction-template.

28 Adelaide, T.U.o. JBI, critical appriasal tools 2020 [cited 2021 May 11]; Available from: https://jbi.global/critical-appraisal-tools.

29 PT Morley 2009 Evidence evaluation worksheets: the systematic reviews for the evidence evaluation process for the 2010 International Consensus on Resuscitation Science Resuscitation 807719721

30 FE Babl 2006 Inhaled methoxyflurane as a prehospital analgesic in children Emerg Med Austral 18440410

31 B Lord PA Jennings K Smith 2016 The epidemiology of pain in children treated by paramedics Emerg Med Austral 28331924

32 APH Karlsen 2014 Safety of intranasal fentanyl in the out-of-hospital setting: a prospective observational study Ann Emerg Med 636699703

33 PA Jennings B Lord K Smith 2015 Clinically meaningful reduction in pain severity in children treated by paramedics: a retrospective cohort study Am J Emerg Med 3311158790 
34 IG Jacobs 2010 Health effects of patients given methoxyflurane in the pre-hospital setting: a data linkage study Open Emerg Med J 3713

35 PP Bredmose 2009 Pre-hospital use of ketamine in paediatric trauma Acta Anaesthesiologica Scandinavica 534543545

36 JC Bendall PM Simpson PM Middleton 2011 Effectiveness of prehospital morphine, fentanyl, and methoxyflurane in pediatric patients Prehosp Emerg Care 152158165

37 AP Murphy 2017 Intranasal fentanyl for the prehospital management of acute pain in children Eur J Emerg Med 246450454

38 G Savoia 2015 Italian Intersociety Recommendations on pain management in the emergency setting (SIAARTI, SIMEU, SIS 118, AISD, SIARED, SICUT, IRC) Minerva Anestesiol 812205225

39 M Borland 2007 A randomized controlled trial comparing intranasal fentanyl to intravenous morphine for managing acute pain in children in the emergency department Ann Emerg Med 493335340

40 Murphy A, et al. Intranasal fentanyl for the management of acute pain in children. Cochrane Database Syst Rev. 2014(10).

41 ML Borland I Jacobs G Geelhoed 2002 Intranasal fentanyl reduces acute pain in children in the emergency department: a safety and efficacy study Emerg Med 143275280

42 F Coffey 2014 STOP!: a randomised, double-blind, placebo-controlled study of the efficacy and safety of methoxyflurane for the treatment of acute pain Emerg Med J 318613618

43 Yousefifard $\mathrm{M}$, et al. Pre-hospital pain management; a systematic review of proposed guidelines. Arch Acad Emerg Med. 2019;7(1).

44 D Braude M Richards 2004 Appeal for fentanyl prehospital use Prehosp Emerg Care 84441

45 A Fabbri 2020 Role of inhaled methoxyflurane in the management of acute trauma pain J Pain Res 131547

46 C Rickard 2007 A randomized controlled trial of intranasal fentanyl vs intravenous morphine for analgesia in the prehospital setting Am J Emerg Med 258911917

47 V Bounes $2008 \mathrm{Is}$ there an ideal morphine dose for prehospital treatment of severe acute pain? A randomized, double-blind comparison of 2 doses Am J Emerg Med 262148154

48 JE Svenson MK Abernathy 2007 Ketamine for prehospital use: new look at an old drug Am J Emerg Med 258977980

49 K Porter 2004 Ketamine in prehospital care Emerg Med J 213351354

50 P Jennings P Cameron S Bernard 2011 Ketamine as an analgesic in the pre-hospital setting: a systematic review Acta anaesthesiologica scandinavica 556638643

51 ST Verghese RS Hannallah 2010 Acute pain management in children J Pain Res 3105

52 RD Blondell M Azadfard AM Wisniewski 2013 Pharmacologic therapy for acute pain Am Family Physic 8711766772

53 WB Crandell SG Pappas A Macdonald 1966 Nephrotoxicity associated with methoxyflurane anesthesia J Am Soc Anesthesiol 27591

54 PTomlin 1965 Methoxyflurane Bt J Anaesthesia 379706709

55 N Vadivelu 2016 Role of ketamine for analgesia in adults and children J Anaesthesiol Clin Pharmacol 323298

56 AM Burnett 2012 The emergency department experience with prehospital ketamine: a case series of 13 patients Prehosp Emerg Care 164553 559

57 PM Lago 2003 Analgesia and sedation in emergency situations and in the pediatric intensive care unit Jornal de pediatria 79 S223 S230

\section{Publisher's Note}

Springer Nature remains neutral with regard to jurisdictional claims in published maps and institutional affiliations.

Ready to submit your research? Choose BMC and benefit from:

- fast, convenient online submission

- thorough peer review by experienced researchers in your field

- rapid publication on acceptance

- support for research data, including large and complex data types

- gold Open Access which fosters wider collaboration and increased citations

- maximum visibility for your research: over $100 \mathrm{M}$ website views per year

At $\mathrm{BMC}$, research is always in progress.

Learn more biomedcentral.com/submissions 